\title{
MONOTONE, HIGHER-ORDER ACCURATE, MULTI-DIMENSIONAL UPWINDING
}

\author{
B. Koren and H.T.M. van der Maarel
}

CWI, P.O. Box 4079, 1009 AB Amsterdam, The Netherlands

\section{Introduction}

Multi-D upwind discretizations for the steady Euler equations are studied, with the emphasis on both accuracy and solvability. The multi-D upwind schemes to be considered here use neither decoupling of the Euler equations as in $[2,7]$, nor rotated fluxes as in $[1,5]$. The schemes are only based on a rotated interpolation of the left and right cell face states. Per cell face, just as with grid-aligned upwind schemes, only a single numerical flux is computed: the one normal to the cell face. First-order accurate versions of these rotated-interpolation schemes have already been investigated in [3]. In the present paper we make an extension to second-order accuracy for a first-order accurate scheme from [3], which has good solvability properties. We try to maintain these properties.

\section{Extension to monotone, second-order accuracy}

We consider the linear, scalar, 2-D model equation

$$
a \frac{\partial u}{\partial x}+b \frac{\partial u}{\partial y}=0, \quad 0 \leq \theta \equiv \arctan \left(\frac{b}{a}\right) \leq \frac{\pi}{2} .
$$

The extension to second-order accuracy is made for the first-order accurate, four-point compact, rotated-interpolation scheme, with the stencil:

$$
\frac{1}{a+b}\left[\begin{array}{ccc}
\cdot & \cdot & \cdot \\
-a^{2} & a^{2}+a b+b^{2} & \cdot \\
-a b & -b^{2} & \cdot
\end{array}\right], \quad 0 \leq \theta \leq \frac{\pi}{2} .
$$

Note that the scheme is nine-point compact for the entire $\theta$-range $[0,2 \pi]$. The scheme is differentiable, also at the angles where it switches $\left(\theta=0, \frac{\pi}{2}, \pi, \frac{3 \pi}{2}\right)$. Further, the scheme's crosswind diffusion is significantly lower than that of the standard, gridaligned, first-order accurate upwind scheme, whereas it is still positive [3]. If we stick to nine-point schemes which use nearest points only, the natural second-order accurate extension of scheme (2) is the central, rotated-interpolation scheme with stencil:

$$
\frac{1}{a+b}\left[\begin{array}{ccc}
\cdot & \frac{1}{2} b^{2} & \frac{1}{2} a b \\
-\frac{1}{2} a^{2} & \cdot & \frac{1}{2} a^{2} \\
-\frac{1}{2} a b & -\frac{1}{2} b^{2} & \cdot
\end{array}\right], \quad 0 \leq \theta \leq \frac{\pi}{2} .
$$


To make scheme (3) monotone, while maintaining second-order accuracy, we first introduce a non-smooth limiter. Next, to even allow successful application of a multigrid-Newton method, we also introduce a smooth limiter. To derive both limiters, consider the following blend of schemes (2) and (3):

$$
\begin{aligned}
& \frac{1}{a+b}\left[\begin{array}{ccc}
-\left(1-\omega\left(r_{\text {hor }}\right)\right) a^{2} & \left(1-\omega\left(r_{\text {hor }}\right)\right) a^{2}+\left(1-\omega\left(r_{\text {skew }}\right)\right) a b+\left(1-\omega\left(r_{\text {ver }}\right)\right) b^{2} & \cdot \\
-\left(1-\omega\left(r_{\text {skew }}\right)\right) a b & -\left(1-\omega\left(r_{\text {ver }}\right)\right) b^{2}
\end{array}\right]+ \\
& \frac{1}{a+b}\left[\begin{array}{ccc}
\cdot & \omega\left(r_{\text {ver }}\right) \frac{1}{2} b^{2} & \omega\left(r_{\text {skew }}\right) \frac{1}{2} a b \\
-\omega\left(r_{\text {hor }}\right) \frac{1}{2} a^{2} & \cdot & \omega\left(r_{\text {hor }}\right) \frac{1}{2} a^{2} \\
-\omega\left(r_{\text {skew }}\right) \frac{1}{2} a b & -\omega\left(r_{\text {ver }}\right) \frac{1}{2} b^{2} & \cdot
\end{array}\right], \quad 0 \leq \omega(r) \leq 1, \quad 0 \leq \theta \leq \frac{\pi}{2},
\end{aligned}
$$

with $\omega(r)$ the limiter function and $r_{\text {hor }}, r_{\text {skew }}$ and $r_{\text {ver }}$ the following ratios of consecutive solution gradients:

$$
\left(\begin{array}{c}
\left(r_{\text {hor }}\right)_{i, j} \\
\left(r_{\text {skew }}\right)_{i, j} \\
\left(r_{\text {ver }}\right)_{i, j}
\end{array}\right)=\left(\begin{array}{c}
\left(u_{i+1, j}-u_{i, j}\right) /\left(u_{i, j}-u_{i-1, j}\right) \\
\left(u_{i+1, j+1}-u_{i, j}\right) /\left(u_{i, j}-u_{i-1, j-1}\right) \\
\left(u_{i, j+1}-u_{i, j}\right) /\left(u_{i, j}-u_{i, j-1}\right)
\end{array}\right)
$$

Note that in the monotonicity theory of e.g. Sweby [8], a blend is taken of the gridaligned, first-order accurate upwind scheme and the grid-aligned, second-order accurate, fully one-sided upwind scheme (i.e. the $\kappa=-1$-scheme in terms of Van Leer [4]). In the present paper we follow more the lines of Yee's symmetric TVD approach [9]. However, the novelty is that we also consider a ratio of consecutive solution gradients which is not grid-aligned $\left(r_{\text {skew }}\right)$. A second difference with the existing, symmetric TVD approach is that we consider ratios of consecutive solution gradients which are defined per cell center and not per cell face. (The pursuit of compactness requires a cell-center approach, instead of a cell-face approach.) An apparent drawback of this cell-centered way to compute the ratios of consecutive solution gradients, is loss of conservation. However, it can be easily shown that this loss is only $\mathcal{O}\left(h^{2}\right)$.

We proceed by deriving the limiters. With (4b), blended scheme (4a) can be cast into the four-point compact form

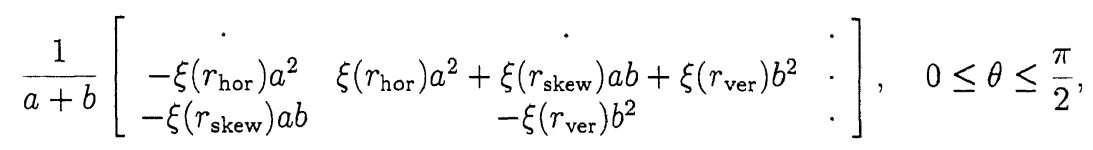

where

$$
\xi(r)=1+\frac{1}{2} \omega(r)(r-1)
$$

The coefficients in $(5 \mathrm{a})$ are then required to satisfy the rules of positivity and finity $(0 \leq \xi(r)<\infty)$. Together with the requirement $\omega(r) \in[0,1]$, this may be combined to

$$
\left(\begin{array}{c}
0 \\
-\lambda
\end{array}\right) \leq\left(\begin{array}{c}
\omega(r) \\
r \omega(r)
\end{array}\right) \leq\left(\begin{array}{c}
1 \\
m
\end{array}\right), \quad 0 \leq m<\infty .
$$

The accuracy requirements that we impose are

$$
\left(\begin{array}{c}
\omega(1) \\
\omega^{\prime}(1)
\end{array}\right)=\left(\begin{array}{l}
1 \\
0
\end{array}\right)
$$


A proper monotonicity function which satisfies (6) and (7) is e.g.

$$
\omega(r)=\max \left[\min \left(\frac{-1}{r}, 1\right), \min \left(1, \frac{m}{r}\right)\right], \quad 1<m<\infty,
$$

where $m$ can be chosen arbitrarily in the indicated range. For all $r$, limiter (8) simply is the upper bound of the monotonicity domain. The limiter is such that the resulting scheme is centered scheme (3) for all $r$ in the range $[-1, m]$. (By choosing a higher value of $m$, the limited scheme equals scheme (3) over a wider range of $r$.) In Fig. 1a we depict the limiter and monotonicity domain, which result for $m=2$. Because of its non-differentiability, limiter (8) is not suited for a Newton-type solution method.

The second limiter to be presented now is differentiable. We assume the form

$$
\omega(r)=\frac{1}{\alpha r^{2}+\beta r+\gamma},
$$

where $\alpha, \beta, \gamma \in \mathbb{R}$ are unknown constants. By imposing requirements (6) and (7), we get the limiter

$$
\omega(r)=\frac{4 m(m-1)}{r^{2}-2 r+4 m(m-1)+1}, \quad 1<m \leq 2,
$$

where $m$ can be chosen freely in the indicated range. (For $m>2$ the limiter no longer satisfies $-1 \leq r \omega(r)$ for all $r$.) In Fig. 1b we depict a simple example of the present smooth limiter $(4 m(m-1)=1)$, together with the corresponding, most tight-fitting monotonicity domain.

\section{Numerical results}

Although the accurate, non-smooth limiter (8) is not suited for our purposes (a multigrid-Newton method for the Euler equations), to have a reference for smooth limiter (10), we still examine limiter (8)'s performance for the known, rotating cone problem (see e.g. [6]). This problem is governed by a linear, scalar convection equation on a square domain, where the wind field is a given, steady solid-body rotation around the square's center. The exact solution on a uniform, $129 \times 129$ finite-volume grid is given in Fig. 2a. Applying an explicit, fourth-order accurate, four-stage Runge-Kutta scheme (with the time step sufficiently small to ensure that the time discretization error is negligible with respect to the space discretization error), we obtain the numerical results given in Figs. $2 \mathrm{~b}-2 \mathrm{~d}$. Non-smooth limiter (8), with $m=2$, appears to yield monotonicity (Fig. 2c), without reduction of the solution accuracy to that of the rotated, first-order accurate scheme (Fig. $2 \mathrm{~b}$ ). In Fig. $2 \mathrm{~d}$ we present the solution obtained by smooth limiter $(10)$, with $4 m(m-1)=1$. It appears that this smoothlimiter-solution is only slightly less accurate than the non-smooth-limiter solution given in Fig. 2c.

Next, smooth limiter $(10)$, with $4 m(m-1)=1$, is applied to a steady, 2-D Euler flow with oblique contact discontinuity. The exact solution on a uniform, $32 \times 32$ finite-volume grid is given in Fig. 3a; the numerical results are given in Figs. 3b and 3c. Just as for the rotating cone problem, also for this Euler flow problem, the smooth limiter appears to lead to monotonicity (Fig. 3c), without reduction of the solution accuracy to that of the rotated, first-order accurate scheme (Fig. 3b). 
It can be concluded that compact, monotone, second-order accurate, rotatedinterpolation schemes: (i) are easily implemented, and (ii) may be solved directly by multigrid-Newton iteration.

\section{References}

[1] S.F. DAvis, 'A rotationally biased upwind difference scheme for the Euler equations', J. Comput. Phys., 56, 65-92 (1984).

[2] Ch. Hirsch, C. LaCor and H. Deconinck, 'Convection algorithms based on a diagonalization procedure for the multidimensional Euler equations', AIAA Paper 87-1163 (1987).

[3] B. Koren AND P.W. Hemker, 'Multi-D upwinding and multigridding for steady Euler flow computations', Proceedings of the Ninth GAMM Conference on Numerical Methods in Fluid Mechanics, Lausanne, 1991, Notes on Numerical Fluid Mechanics (A. Rizzi and I.L. Ryhming, eds.), Vieweg, Braunschweig (to appear).

[4] B. VAN LEER, 'Upwind-difference methods for aerodynamic problems governed by the Euler equations', Proceedings of the 15th AMS-SIAM Summer Seminar on Applied Mathematics, Scripps Institution of Oceanography, 1983, Lectures in Applied Mathematics, 22, Part 2, 327-336 (B.E. Engquist, S.J. Osher and R.C.J. Somerville, eds.), American Mathematical Society, Providence, Rhode Island (1985).

[5] D.W. Levy, K.G. Powell and B. van Leer, 'An implementation of a gridindependent upwind scheme for the Euler equations', AIA A paper 89-1931 (1989).

[6] S.A. Orszag, 'Numerical simulation of incompressible flows within simple boundaries: accuracy', J. Fluid Mech., 49, 75-112 (1971).

[7] P.L. RoE, 'Discrete models for the numerical analysis of time-dependent multidimensional gas dynamics', J. Comput. Phys., 63, 458-476 (1986).

[8] P.K. Sweby, 'High resolution schemes using flux limiters for hyperbolic conservation laws', SIAM J. Numer. Anal., 21, 995-1011 (1984).

[9] H.C. YEE, 'Construction of explicit and implicit symmetric TVD schemes and their applications', J. Comput. Phys., 68, 151-179 (1987).

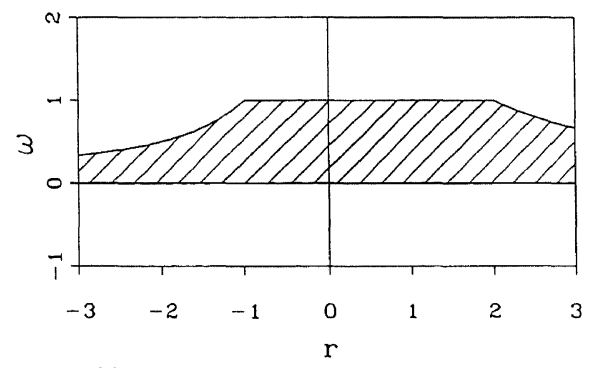

a. Non-smooth limiter $\omega(r)=\max \left[\min \left(\frac{-1}{r}, 1\right), \min \left(1, \frac{2}{r}\right)\right]$.

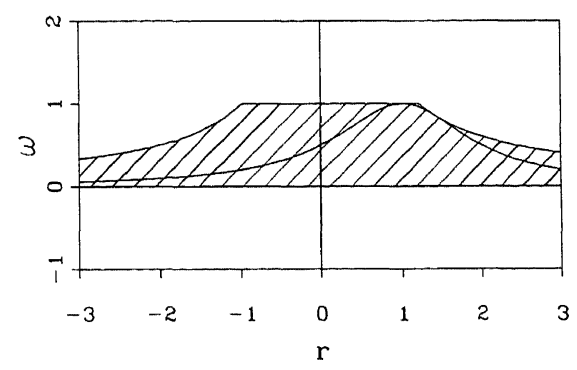

b. Smooth limiter $\omega(r)=\frac{1}{(r-1)^{2}+1}$.

Figure 1: Non-smooth limiter, smooth limiter and monotonicity domains. 


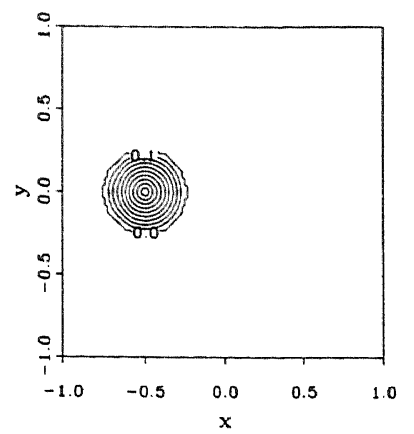

a. Exact.

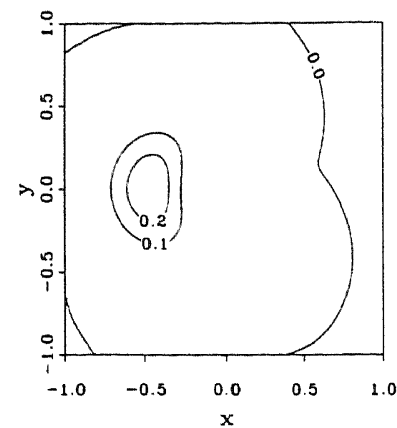

b. First-order scheme.

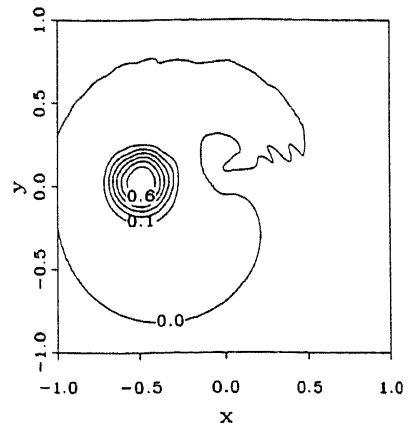

c. Higher-order scheme, with limiter from Fig. 1a.

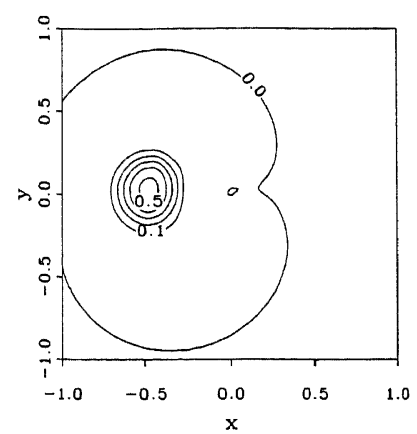

d. Higher-order scheme, with limiter from Fig. 1b.

Figure 2: Solutions rotating cone problem, after a single rotation.

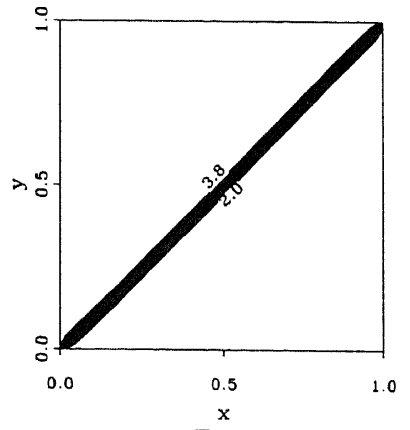

a. Exact.

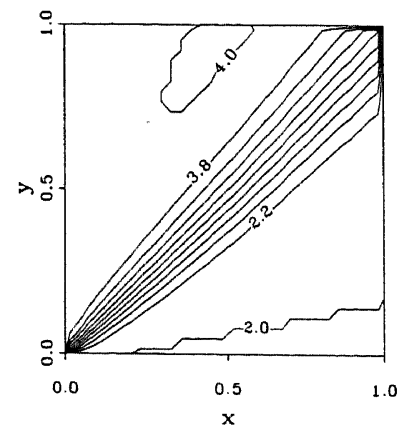

b. First-order scheme.

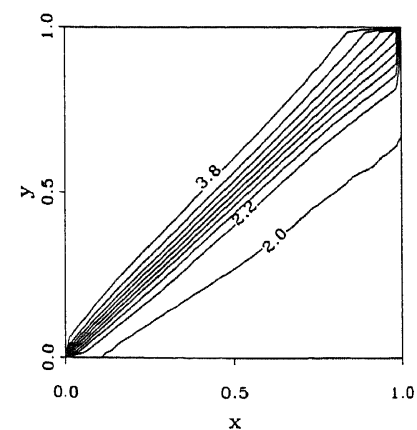

c. Higher-order scheme, with limiter from Fig. $1 b$.

Figure 3: Mach number distributions oblique contact discontinuity. 